
\title{
25 Research Square \\ Effect of the first mating experience on mating behaviors and brain gene expression in male medaka
}

Masahiro Daimon

Okayama University

Takafumi Katsumura

Kitasato University

Hirotaka Sakamoto

Okayama University

Satoshi Ansai

Tohoku University

Hideaki Takeuchi ( $\nabla$ hideaki.takeuchi.a8@tohoku.ac.jp )

Tohoku University

Research Article

Keywords:

Posted Date: February 25th, 2022

DOI: https://doi.org/10.21203/rs.3.rs-1368136/v1

License: (9) This work is licensed under a Creative Commons Attribution 4.0 International License. Read Full License 


\section{Abstract}

The first mating (sexual) experience leads to the maturation of male mating behavior across species (insects, fish, rodents). Here, we investigated whether the first mating experience leads to maturation of male mating behavior in medaka using repeated mating tests. In "naïve" (sexually inexperienced) males after the first mating experience, the latency to mate with the same female partner was significantly decreased, whereas when the partner was swapped, the latency to mate was not affected. These findings suggest that repeated matings ( 3 times) enhanced male mating activity for the familiarized female, but not for an unfamiliarized female. In "experienced" (> 7 matings) males, repeated matings (3 times) with the same partner did not influence the latency to mate, suggesting that multiple matings ( $>7$ times) abolish the mate preference of naïve males. Furthermore, we identified 10 highly and differentially expressed genes after the mating experience in the brains of the post-naïve males, and revealed 3 genes that are required for a functional cascade of the thyroid hormone system. These findings together suggest that the first mating experience abolishes the preference to mate with a familiarized female via neural maturation triggered by thyroid hormone activation in the medaka brain.

\section{Introduction}

The first mating experience leads to the maturation of male mating behavior across species. For example, in fruit flies (Drosophila melanogaster), the first mating experience in males shortens the latency to the first courtship with a female ${ }^{1}$. Furthermore, in a triangle relationship (naïve male, experienced male, and receptive female), experienced males more frequently exhibit abdomen bends (attempted copulation) than naïve males. In rodents, the first male mating experience decreases the latency to the first intromission (mounting behavior with penis insertion) in mice ${ }^{2,3}$ and rats ${ }^{4}$. In fish species, however, only one published report indicates that the emergence of mate preference depends on the mating experience. Vega-Trejo et al. quantified mate preference in mosquitofish (Gambusia holbrooki) using a 3-chamber test, and demonstrated that the amount of time spent with a novel female is significantly increased by the mating experience, but not by visual and olfactory familiarization ${ }^{5}$.

In rodents, the behavioral maturation triggered by the first mating experience is associated with brain neural maturation. The number of neurons in the olfactory bulbs and the density of mushroom spines in the medial preoptic area ( $\mathrm{MPOA}$ ) are increased by the first mating experience in mice ${ }^{2,6}$. In contrast, the mushroom spine density in the MPOA decreases and the expression of $v g f$, which encodes the neuropeptide precursor VGF in the MPOA, affects behavioral maturation (shortening mating latency) following the mating and ejaculation experience in male rats ${ }^{4,7}$. We recently reported that the expression of gastrin-releasing peptide and oxytocin receptors is increased in the spinal ejaculation generator in the lumbosacral cord after the first mating experience with an ejaculation in male rats ${ }^{8}$. Furthermore, the first mating experience also decreases neuronal activity in the center part of the MPOA, suggesting that the first mating experience reconstructs the neural network associated with the male mating behavior ${ }^{9}$. No 
studies to date have revealed whether the neural/molecular mechanisms underlying behavioral changes dependent on the first mating are conserved among vertebrates.

To address this question, we used medaka fish (Oryzias latipes) in the present study. There are many advantages to using medaka fish for studies of mating behavior. First, medaka mating behavior comprises several steps (approach, courtship display, wrapping, and spawning), which allow for quantification of male mating activity under laboratory condition ${ }^{10}$. Second, as the female reproductive cycle is $24 \mathrm{~h}$, the same female ready to spawn can be used for mating tests every morning ${ }^{11}$. Third, medaka is a model animal for molecular genetics and state-of-the-art molecular genetic techniques are available. Here, we show that the first male mating experience abolishes the mating preference for familiarized females in medaka fish. Furthermore, transcriptome studies suggested the possible involvement of the thyroid system in the behavioral changes dependent on the male mating experience in medaka fish.

\section{Methods}

\section{Ethics statement}

All the methods in this study were carried out in accordance with relevant guidelines and regulations. The work in this paper was conducted using protocols specifically approved by the Animal Care and Use Committee of Okayama University (permit number: OKU-2015467). All surgery was performed under deep anesthesia using ice, and all efforts were made to minimize suffering following the NIH Guide for the Care and Use of Laboratory Animals Fish and breeding conditions. The study was carried out in compliance with the ARRIVE guidelines (https://arriveguidelines.org/arrive-guidelines).

\section{Animal maintenance}

All fish (Oryzias latipes, d-rR strain) were bred in our laboratory. Fish larvae were fed Paramecium or small pellet foods (Medaka no Mai Next, Kyorin, Japan), juveniles were fed small pellet foods (Medaka no Mai Next), and adult medaka were fed pellet foods (Otohime B2, Nisshin-marubeni, Japan) a few times a day. Juvenile and adult fish were fed brine shrimp once a day. Medaka were maintained in groups in plastic aquariums ( $13 \mathrm{~cm} \times 19 \mathrm{~cm} \times 12 \mathrm{~cm}$ height) or polypropylene containers (48 cm x $36 \mathrm{~cm} \times 20 \mathrm{~cm}$ height). The water temperature was maintained at $24-28^{\circ} \mathrm{C}$ with white LED lights (Eco-slim, OHM ELECTRIC INC, Japan) for $14 \mathrm{~h}$ per day (08:00-22:00). At another laboratory (Okayama University, Japan), all medaka were maintained in plastic aquariums $(13 \mathrm{~cm} \times 19 \mathrm{~cm} \times 12 \mathrm{~cm}$ height); the larvae were fed Paramecium, juveniles were fed small pellet foods (Hikari lab., Meito system, Japan), and adult medaka were fed flake foods (TetraMin, Tetra, Germany) a few times a day and brine shrimp once a day.

\section{Animal preparation for mating tests}

Adult male ( $>5$ months of age) and female ( $>3$ months of age) fish were used for this experiment. To prepare "naïve males", we separated sexually immature males from females 1-2 months after hatching. 
We determined their sexes based on the body color difference ${ }^{12}$ and fin shape. We used sexually matured females that had spawned fertilized eggs continuously for at least 3 days as sexually-matured ready-tospawn eggs. In the present study, we defined "naïve" males as sexually inexperienced and "experienced males" as adult males that had mated with females more than 7 times.

\section{Mating test using fixed pairs}

The mating test was performed as previously described ${ }^{13}$. To separate the male from the female, a plastic cup (CE-300, Kenis, Japan, $37 \mathrm{~mm}$ [radius] x $90 \mathrm{~mm}$ [height]) with white opaque paper was used from the night to the next morning (16:00-10:00). To habituate the fish to this experimental condition before starting the mating test, males were placed in the cup overnight at least 3 times. The day before the mating test, the opaque plastic cup with the naïve or experienced male was placed into a tank containing a female. The next morning, a male was released toward the tank containing the female, which allowed them to begin their mating behavior (9:30-10:30). We recorded their mating behavior for 15 min using a Web camera (BSW200MKB, Buffalo, Japan). We repeated the mating tests for 7 days (times) using the same pairs. If a female did not spawn at least once over the 7 days, we excluded all data from the analysis. We manually measured the timing of the courtship display (male quick-circle dance), wrapping (crossing each body), wrapping rejection (wrapping with no spawning), and spawning by viewing the video, and calculated the latency to mate (period from releasing the male to the wrapping with spawning), the latency to the first courtship display, and the latency to mate from the first courtship. Experienced males who had mated with females more than 7 times were used for the same experiments as a control.

\section{Mating test using swapped pairs}

To determine whether the mating experience with the same partner was essential for changing the mating behavior, we performed the mating test using fixed and swapped pairs using naïve males continuously for 3 days (times). The procedure was the same as for the mating test described above.

\section{Statistical analysis}

Statistical analysis was run by $\mathrm{R}$ (version 4.0.5) with generalized linear mixed models (GLMMs) by the "glmer" function in the package Ime4 (version 1.1-27) to reveal whether the number of matings 
(experience) affected medaka mating behavior. The gamma distribution (latency to mate, latency to the first courtship display, and latency to mate from the first courtship) and Poisson distribution (number of courtship displays, wrappings, wrapping rejections, and courtship from courtship [Cou $\rightarrow$ Cou], wrapping from courtship [Cou $\rightarrow$ Wra], courtship from wrapping rejection [W.R $\rightarrow$ Cou], and wrapping rejection from wrapping [Wra $>$ W.R]) with a log link function were used for each statistical analysis. Individual male and female (only in swapped pairs) identification numbers were included as random intercepts. We constructed both full models including mating times as an explanatory variable and null models with no explanatory variable, and then compared the models using the likelihood ratio test. To select the model with the best predictability, we compared the Akaike Information Criterion (AIC) between the 2 models. When the likelihood ratio test indicated a significant effect of the mating times $(P<0.05)$, adjusted $P$ values calculated using the emmeans package (version 1.6.1) with the Tukey method are shown for post hoc test.

\section{RNA-Seq and Data Analysis}

Whole brains with the pituitary were collected from naïve males with no mating experience and post-naïve males (after 2 mating experiences) in the morning just before the third mating. The dissected brains were stabilized with RNA-later (Thermo Fisher Scientific, USA) until the extraction steps. Total RNAs were extracted using TRI Reagent (Cosmo Bio, Japan) and then purified using an RNeasy plus mini kit (Qiagen, Germany). RNA extraction solutions (same concentrations) from 3 individuals were included as 1 sample for RNA-seq (each sample size was 2). All library preparations and sequencing were outsourced to a company (GENEWIZ, Japan). The cDNA libraries were prepared using a NEBNext Ultra RNA Library Prep Kit for Illumina (New England Biolabs, USA). The libraries were multiplexed and loaded on an Illumina HiSeq X Ten ( 2 x 150 bp, Illumina, USA). Adapter sequences were trimmed using Cutadapt ${ }^{14}$ (v1.9.1). Reference genome sequences and gene model annotation files of medaka (Annotation NO: ASM223467v1) were used. Second, HISAT2 ${ }^{15}$ was used to index the reference genome sequence. Finally, clean data were aligned to the reference genome via software HISAT2 (v2.0.1). We used edgeR ${ }^{16}$ (v3.4.6) for differential expression analysis. After normalization with the TMM method, differential expressed genes were identified with an FDR adjusted $p$ value $<0.01$ and |Fold change| $>2$.

\section{Results}

\section{The first mating experience in males decreased the latency to mate}

To investigate how the first mating experience in males could influence mating behavior in medaka fish, we performed mating tests using naïve males and experienced males who had mated with females more than 7 times. To prepare naïve males, we separated juvenile males into groups and bred them without any females until performing the mating tests. We also prepared sexually mature females (>3 months after hatching) for the mating test. The mating tests were carried out using 11 fixed pairs for 7 days ( 7 
times) (Fig. 1A, 1B). The latency to mate was defined as the interval from "releasing the male" to "crossing with spawning". To evaluate the effect of the first mating experience on the latency to mate, we used a GLMM, which revealed that the latency of naïve males significantly decreased after the first mating experience and biased the distribution for 5 days, but not for 7 days (Fig. 1C, Table S1, GLMM; day 1 vs day 3 estimate \pm s.e. $=0.5094 \pm 0.192$, $z$.ratio $=2.646, P=0.0406$, day 1 vs day 5 estimate \pm s.e. $=$ $0.8494 \pm 0.189$, z.ratio $=4.488, P=<0.0001$, day 1 vs day7 estimate \pm s.e. $=0.4231 \pm 0.219$, z.ratio $=1.934$, $P=0.2137$, day 3 vs day 5 estimate \pm s.e. $=0.3401 \pm 0.191$, z.ratio $=1.781, P=0.2825$, day 3 vs day 7 estimate \pm s.e. $=-0.0862 \pm 0.217$, z.ratio $=0.398, P=0.978$, day 5 vs day7 estimate \pm s.e. $=-0.4263 \pm$ 0.218 , $z$.ratio $=-1.951, P=0.2067)$. In experienced males, on the other hand, the mating experience had no significant effect on the latency to mate (Fig. 1C, Table S1). Together, these findings indicated that the male mating experience influenced medaka mating behavior only in naïve males. We confirmed that these tendencies were reproduced in experiments performed at a different laboratory (Fig. S1, Table S2, S3). Therefore, we concluded that the first mating experience in naïve males decreased the latency to mate.

\section{The first mating experience shortened the latency to a first courtship display}

We further examined which behavioral component could influence the latency to mate in naïve males in repeated mating tests. First, we counted the number of courtship displays and measured the latency to the first courtship display, revealing that the latency to the first courtship display was significantly decreased only in naïve males, and not in experienced males (Fig. 2A, Tables S1, GLMM; day 1 vs day3 estimate \pm s.e. $=0.0973 \pm 0.189$, . ratio $=0.515, P=0.9555$, day 1 vs day 5 estimate \pm s.e. $=0.6015 \pm 0.186$, $z$.ratio $=3.229, P=0.0068$, day 1 vs day 7 estimate \pm s.e. $=0.7165 \pm 0.201$, $z$.ratio $=3.562, P=0.0021$, day 3 vs day 5 estimate \pm s.e. $=0.5043 \pm 0.171$, z.ratio $=2.951, P=0.0167$, day 3 vs day 7 estimate \pm s.e. $=0.6192$ \pm 0.198 , z.ratio $=3.135, P=0.0093$, day 5 vs day7 estimate \pm s.e. $=0.115 \pm 0.196$, z.ratio $=0.586, P=$ 0.963). On the other hand, the number of courtship displays did not significantly change in either naïve or experienced males (Fig. 2B, Table S1). Next, we compared the latency to mate from the first courtship display, which negatively correlated with the degree of female receptivity ${ }^{12-14}$. The latency was significantly decreased only in naïve males (Fig. 2C, Table 1, GLMM; day1 vs day3 estimate \pm s.e. $=$ $1.4486 \pm 0.331$, z.ratio $=4.379, P=0.0001$, day 1 vs day 5 estimate \pm s.e. $=1.5358 \pm 0.346$, z.ratio $=4.44, P$ $=0.0001$, day 1 vs day 7 estimate \pm s.e. $=0.3013 \pm 0.4$, z.ratio $=0.754, P=0.875$, day 3 vs day 5 estimate \pm s.e. $=0.872 \pm 0.326$, z.ratio $=0.268, P=0.9933$, day3 vs day7 estimate \pm s.e. $=-1.1472 \pm 0.377$, z.ratio $=$ $3.041, P=0.0126$, day 5 vs day7 estimate \pm s.e. $=-1.2344 \pm 0.385$, $z$.ratio $=-3.207, P=0.0073)$, and strongly suggested that the female mating experience with the naïve males enhanced female receptivity. Furthermore, we analyzed other behavioral elements (wrappings and wrapping rejections), and found no significant change between the number of the 2 behavioral elements and the number of mating in either naïve or experienced males (Fig. S3, Table S1). Next, we analyzed behavioral transitions in the mating test, and found that the number of courtship displays significantly decreased between day 1 vs day 3 and day 1 vs day 5 in experienced males (Fig. S5, Tables S1, GLMM; day1 vs day3 estimate \pm s.e. $=0.636 \pm$ 0.234 , $z$.ratio $=2.717, P=0.0333$, day1 vs day 5 estimate \pm s.e. $=0.820 \pm 0.251, z$.ratio $=3.26, P=0.0061$, 
day 1 vs day7 estimate \pm s.e. $=0.42 \pm 0.223$, z.ratio $=1.881, P=0.2361$, day3 vs day5 estimate \pm s.e. $=$ $0.184 \pm 0.283$, z.ratio $=0.649, P=0.9158$, day3 vs day7 estimate \pm s.e. $=-0.216 \pm 0.258$, $z$.ratio $=-0.838$, $P=0.8365$, day 5 vs day7 estimate \pm s.e. $=-0.400 \pm 0.273$, z.ratio $=-1.464, P=0.4592)$. In addition, we confirmed that these tendencies were reproduced in experiments performed at a different laboratory (Fig. S4, S6, Tables S2, S3). Accordingly, we revealed that the first mating experience shortened the latency to the first courtship display only in naïve males.

Table 1

RNA-seq results. List of differentially expressed genes (DEGs) that were more highly expressed in post-naïve samples (2 matings) than in naïve samples. Genes are ordered from high to low expression using FPKM values as an index.

\begin{tabular}{|c|c|c|c|c|c|}
\hline & \multirow[t]{2}{*}{ gene } & \multirow[t]{2}{*}{$\log _{2}|\mathrm{FC}|$} & \multirow[t]{2}{*}{ FDR } & \multirow{2}{*}{$\begin{array}{l}\text { FPKM } \\
\text { (naïve) }\end{array}$} & \multirow{2}{*}{$\begin{array}{l}\text { FPKM } \\
\text { (post-naïve) }\end{array}$} \\
\hline & & & & & \\
\hline 1 & fkbp5 & 1.89 & $1.010 \mathrm{E}-18$ & 23.68 & 87.57 \\
\hline 2 & tshba & 2.72 & 3.320E-39 & 6.99 & 45.93 \\
\hline 3 & hapln2 & 1.12 & 4.760E-05 & 19.22 & 41.74 \\
\hline 4 & LOC101155558 & 1.40 & $3.284 \mathrm{E}-03$ & 11.44 & 30.26 \\
\hline 5 & macrosialin & 1.04 & 1.990E-05 & 14.57 & 29.91 \\
\hline 6 & stat1a & 1.13 & $5.290 \mathrm{E}-07$ & 10.18 & 22.34 \\
\hline 7 & lgals17 & 1.76 & 1.730E-06 & 4.97 & 16.87 \\
\hline 8 & dio2 & 1.05 & $9.331 \mathrm{E}-04$ & 7.30 & 15.10 \\
\hline 9 & Igals3bp & 1.39 & 5.390E-07 & 5.77 & 15.10 \\
\hline 10 & klf9 & 1.10 & 4.730E-08 & 6.24 & 13.36 \\
\hline
\end{tabular}

The behavioral change in naïve males occurred only in fixed pairs

The first male mating experience mainly influenced the latency to mate and the latency to the first courtship display in fixed pairs in 7 continuous mating tests. Here we examined whether this effect was specific for fixed pairs. To compare mating behavior between fixed pairs and swapped pairs, we performed mating tests for 3 days (Fig. 3A, each $n=8$ ). The latency to mate and the latency to the first courtship display were significantly decreased in the fixed group (GLMM; day1 vs day2 estimate \pm s.e. $=$ $0.205 \pm 0.00818$, $z$.ratio $=2.5, P=0.0332$, day 1 vs day3 estimate \pm s.e. $=0.345 \pm 0.00779$, z.ratio $=4.432$, $P<0.0001$, day 2 vs day 3 estimate \pm s.e. $=0.141 \pm 0.0785$, $z$.ratio $=1.791, P=0.1725$ ), but did not change in swapped pairs (Fig. 3B, 3C, Table S4). The latency to mate from the first courtship display (Fig. 3D, Table S4), numbers of other events (Fig. S7, Table S4), and behavioral transitions (Fig. S8, Table S4) did not significantly change in either fixed or swapped pairs. The latency to mate and the latency to the first 
courtship display significantly decreased in repeated behavioral experiments ( 3 times). These findings strongly suggest that the naïve males recognized the first mating partner and thus the latency to mate and the latency to the first courtship display were decreased according to the mating experience.

\section{The first mating experience changed gene expression in the male brain}

To evaluate the effect of the first mating experience on the brain gene expression patterns, we compared gene expression profiles using whole brains between the naïve and post-naïve male medaka that had 2 mating experiences (Fig. S9). In the present study, we performed RNA-seq analysis using the whole brain with the pituitary, because in some fish species such as zebrafish and cichlid, social status has significant effects on gene expression at the whole brain level ${ }^{17,18}$. We identified 10 differentially expressed genes (DEGs) that were upregulated by the mating experience and had a greater than 2-fold change, suggesting that the first mating experience could influence brain gene expression in male medaka. We listed the DEGs in descending order of the expression level (FPKM) (Table 1). Interestingly, we found that 3 genes (tshba, dio2, klf9) of the top 10 DEGs were associated with functional expression of the thyroid hormone system (Table 1). tshba encodes a thyroid stimulating hormone that promotes the synthesis and secretion of inactivated thyroid hormone (T4). dio2 encodes type II iodothyronine deiodinase, which converts inactivated thyroid hormone to activated thyroid hormone (T3). klf9 encodes a transcription factor, Krüppel-like factor 9 , which is induced by thyroid hormone (T3) ${ }^{19}$. These findings indicate that the first mating experience changed the gene expression patterns and upregulated thyroid hormone-related genes. In addition, early-life stress exposure increases $f k b p 5$ expression in the brain and FKBP5 regulates glucocorticoid receptor activity ${ }^{20}$. High $f k b p 5$ expression and early-life stress interact to increase anxiety-like behavior mediated by AKT signaling in association with hippocampal synaptic plasticity ${ }^{21}$. Hapln2 (also called Bral1) is essential for formation of the functional extracellular matrix and neuronal conductivity in mice 22 .

\section{Discussion}

The findings of the present study revealed, in medaka fish, that naïve males altered their mating behavior according to their first mating experience. Interestingly, the pattern of behavioral alterations after the first mating in medaka fish was quite different from those of other species such as fruit flies ${ }^{1,23}$, mice $e^{2,3}$ and rats $^{4}$, in which the mating experience increases male sexual motivation, but does not influence mate preference. In medaka fish, repeated matings (3 times) with the same partners decreased the latency to mate in naïve males, but not in experienced males. This finding suggests that naïve medaka males have a mate preference for familiar females, while experienced medaka males do not. In other words, the first mating experience might abolish familiarized mate preference in male medaka. Most animals change their social strategy from the juvenile stage to the adult stage. During the juvenile stage, the social strategy mainly promotes growth and survival, while in the adult stage, the social strategy includes reproductive behaviors to produce more progeny. For example, juvenile guppies tend to form kin groups to achieve an effective transfer and protect against predators ${ }^{24}$, while adult guppy shoals do not maintain 
kin groups to prevent inbreeding ${ }^{25}$. In other fish species, such as humbug damselfish ${ }^{26}$ and three-spot dascyllus ${ }^{27}$, juveniles tend to exhibit a social preference for familiar individuals. In cichlid, juvenile fish kept in a kin group grow faster ${ }^{28}$ and approach a predator for surveillance more often than solitary individuals that do not live in a group ${ }^{29}$. At the adult stage, mosquito fish exhibit a mate preference for unfamiliar mates, which emerges after the first mating experience. To our knowledge, these findings provide the first evidence indicating that the first mating experience abolishes mate preference in any species. Further studies are needed to elucidate why naïve medaka males have a mate preference based on familiarization and why the multiple ( $>7$ times) mating experience abolishes the preference. It is possible that the first mating experience contributes to changes in social strategy during life.

Furthermore, after the mating experience, we observed the upregulation of 3 genes (tshba, dio2, klf9) related to functional expression of the thyroid hormone. Thyroid hormone is required for brain maturation and development ${ }^{30,31}$. Interestingly, in Japanese quail, thyroid hormone is a trigger hormone for the release of gonadotropin-releasing hormone in the brain to mature gonads for reproduction ${ }^{32}$. A relationship between seasonal reproduction and thyroid hormone in the brain is reported across species, including mice ${ }^{33}$ and fish $^{34}$, among seasonally reproductive animals. In this study, sexually mature males were used for the mating test and there was no change of seasonal information (i.e., water temperature and day length remained the same) in the laboratory, but medaka do show seasonal reproductivity ${ }^{35}$, suggesting that the thyroid hormone system is activated in the brain by the first mating experience and not a seasonal change. To our knowledge, there are no reports of thyroid hormone activation triggered by the first mating experience in any species. In addition, $k / f 9$, which is a transcription factor dependent on the thyroid hormone ${ }^{19}$, contributes to dendritic spine maturation in the mouse hippocampus ${ }^{36}$. The first mating experience leads to maturation of mating behavior and shapes male sexual motivation with neural maturation in the $\mathrm{mPOA}$, which is the center of the male mating behavior in rodents ${ }^{37,38}$. Therefore, the first mating experience may mature male mating social behavior with neural maturation according to thyroid hormone levels in male medaka (Fig. 4). Behavioral maturation by the first mating experience in male rodents might also be related to the thyroid hormone system. The center of the mPOA, including the sexually dimorphic nucleus of the preoptic area, is more activated in naïve male rats than in experienced male rats ${ }^{9}$. In addition, knockdown of $v g f$ expression erases the behavioral maturation dependent on the first mating experience ${ }^{4}$. Interestingly, thyroid hormone regulates $v g f$ expression in hamsters ${ }^{39}$, implying that behavioral maturation by the first mating experience in male rodents is also related to the thyroid hormone system. These findings, however, are fragmented and further studies are necessary to elucidate whether the molecular mechanisms associated with male mating maturation dependent on the first mating experience are conserved among vertebrates.

\section{Declarations}

\section{Data availability}


All sequence read data are available from DDBJ (DRA013480) and the data that support the findings of this study are available from the corresponding author, [HT], upon reasonable request.

\section{Competing interests (mandatory)}

The authors declare no competing interests.

\section{Acknowledgements}

We thank the National BioResource Project Medaka for supplying the medaka strains (https://shigen.nig.ac.jp/medaka). This work was supported by the National Institute for Basic Biology Priority Collaborative Research Project 10-104 (to H.T.), 19-347 (to H.T.), and 21-335 (to H.T.); A grant for Joint Research (\#01111904) by the National Institutes of Natural Sciences (to HT); Japan Society for the Promotion of Science (JSPS) KAKENHI Grants $21 \mathrm{H} 04773$ (to S.A. \& H.T.), $20 \mathrm{H} 04925$ (to H.T.), $18 \mathrm{H} 02479$ (to H.T.); The Mitsubishi Foundation Natural Sciences Research (to H.T); Takeda Science Foundation (to H.T); The JST SPRING Grant JPMJSP2114 (to M.D.).

\section{Author contributions}

M. D., H. S. and H. T. designed research; M. D. performed research; M. D., T. K. and S. A. analyzed data; M. D. and H. T. wrote the paper; All authors reviewed manuscript.

\section{References}

1. Dukas, R. Experience improves courtship in male fruit flies. Anim. Behav. 69, 1203-1209 (2005).

2. Jean, A., Bonnet, P., Liere, P., Mhaouty-Kodja, S. \& Hardin-Pouzet, H. Revisiting medial preoptic area plasticity induced in male mice by sexual experience. Sci. Rep. 7, 1-13 (2017).

3. Swaney, W. T., Dubose, B. N., Curley, J. P. \& Champagne, F. A. Sexual experience affects reproductive behavior and preoptic androgen receptors in male mice. Horm. Behav. 61, 472-478 (2012).

4. Maejima, S. et al. VGF in the medial preoptic nucleus increases sexual activity following sexual arousal induction in male rats. Endocrinology 159, 3993-4005 (2018).

5. Vega-Trejo, R., O'Dea, R. E., Jennions, M. D. \& Head, M. L. The effects of familiarity and mating experience on mate choice in mosquitofish, Gambusia holbrooki. Behav. Ecol. 25, 1205-1211 (2014).

6. Velazco-Mendoza, M., Camacho, F. J., Paredes, R. G. \& Portillo, W. The first mating experience induces new neurons in the olfactory bulb in male mice. Neuroscience 396, 166-174 (2019).

7. Nakashima, S., Morishita, M., Ueno, K. \& Tsukahara, S. Region-specific effects of copulation on dendritic spine morphology and gene expression related to spinogenesis in the medial preoptic nucleus of male rats. Psychoneuroendocrinology 108, 1-13 (2019).

8. Oti, T. et al. Sexual Experience Induces the Expression of Gastrin-Releasing Peptide and Oxytocin Receptors in the Spinal Ejaculation Generator in Rats. Int. J. Mol. Sci. 22, 10362 (2021). 
9. Yamaguchi, S., Abe, Y., Maejima, S. \& Tsukahara, S. Sexual experience reduces neuronal activity in the central part of the medial preoptic nucleus in male rats during sexual behavior. Neurosci. Lett. $685,155-159$ (2018).

10. Ono, Y. \& Uematsu, T. Mating Ethogram in Oryzias latipes. Jour. Fac. Sci. Hokkaido Univ. Ser 13, 197202 (1957).

11. Egami N. Effect of artificial photoperiodicity on time of oviposition in the fish, Oryzial latipes. Annot. Zool. Jpn. 27, 57-62 (1954).

12. Yamamoto, T. Artificial induction of functional sex-reversal in genotypic females of the medaka (Oryzias latipes). J. Exp. Zool. 137, 227-263 (1958).

13. Okuyama, T. et al. A neural mechanism underlying mating preferences for familiar individuals in medaka fish. Science. 343, 91-94 (2014).

14. Martin, M. Cutadapt removes adapter sequences from high-throughput sequencing reads. EMBnet.journal 17, 10-12 (2011).

15. Kim, D., Langmead, B. \& Salzberg, S. L. HISAT: a fast spliced aligner with low memory requirements. Nat. Methods 12, 357 (2015).

16. Robinson, M. D., McCarthy, D. J. \& Smyth, G. K. edgeR: a bioconductor package for differential expression analysis of digital gene expression data. Bioinformatics 26, 139 (2010).

17. Oliveira, R. F. et al. Assessment of fight outcome is needed to activate socially driven transcriptional changes in the zebrafish brain. Proc. Natl. Acad. Sci. U. S. A. 113, E654-E661 (2016).

18. Renn, S. C. P., O'Rourke, C. F., Aubin-Horth, N., Fraser, E. J. \& Hofmann, H. A. Dissecting the transcriptional patterns of social dominance across teleosts. Integr. Comp. Biol. 56, 1250 (2016).

19. Denver, R. J. et al. Basic transcription element-binding protein (BTEB) is a thyroid hormone- regulated gene in the developing central nervous system: Evidence for a role in neurite outgrowth. J. Biol. Chem. 274, 23128-23134 (1999).

20. Scharf, S. H., Liebl, C., Binder, E. B., Schmidt, M. V. \& Müller, M. B. Expression and regulation of the Fkbp5 gene in the adult mouse brain. PLoS One 6, e16883 (2011).

21. Criado-Marrero, M. et al. Early life stress and high FKBP5 interact to increase anxiety-like symptoms through altered AKT signaling in the dorsal hippocampus. Int. J. Mol. Sci. 20, 2738 (2019).

22. Bekku, Y. et al. Bral1: Its role in diffusion barrier formation and conduction velocity in the CNS. J. Neurosci. 30, 3113-3123 (2010).

23. Saleem, S., Ruggles, P. H., Abbott, W. K. \& Carney, G. E. Sexual Experience Enhances Drosophila melanogaster Male Mating Behavior and Success. PLoS One 9, e96639 (2014).

24. Piyapong, C. et al. Kin assortment in juvenile shoals in wild guppy populations. Heredity (Edinb). 106, 749-756 (2011).

25. Russell, S. T., Kelley, J. L., Graves, J. A. \& Magurran, A. E. Kin structure and shoal composition dynamics in the guppy, Poecilia reticulata. Oikos 106, 520-526 (2004). 
26. Buston, P. M., Fauvelot, C., Wong, M. Y. L. \& Planes, S. Genetic relatedness in groups of the humbug damselfish Dascyllus aruanus: small, similar-sized individuals may be close kin. Mol. Ecol. 18, 4707-4715 (2009).

27. Bernardi, G., Beldade, R., Holbrook, S. J. \& Schmitt, R. J. Full-sibs in cohorts of newly settled coral reef fishes. PLoS One 7, e44953 (2012).

28. Hesse, S. \& Thünken, T. Growth and social behavior in a cichlid fish are affected by social rearing environment and kinship. Sci. Nat. 101, 273-283 (2014).

29. Hesse, S., Anaya-Rojas, J. M., Frommen, J. G. \& Thünken, T. Social deprivation affects cooperative predator inspection in a cichlid fish. Royal. Soc. Open Sci. 2, 140451 (2015).

30. Ahmed, O. M., El-Gareib, A. W., El-bakry, A. M., Abd El-Tawab, S. M. \& Ahmed, R. G. Thyroid hormones states and brain development interactions. Int. J. Dev. Neurosci. 26, 147-209 (2008).

31. Uchida, K. et al. Thyroid hormone insufficiency alters the expression of psychiatric disorder-related molecules in the hypothyroid mouse brain during the early postnatal period. Sci. Rep. 11, 1-10 (2021).

32. Yamamura, T., Hirunagi, K., Ebihara, S. \& Yoshimura, T. Seasonal morphological changes in the neuro-glial interaction between gonadotropin-releasing hormone nerve terminals and glial endfeet in Japanese quail. Endocrinology 145, 4264-4267 (2004).

33. Ono, H. et al. Involvement of thyrotropin in photoperiodic signal transduction in mice. Proc. Natl. Acad. Sci. U. S. A. 105, 18238-18242 (2008).

34. Nakane, Y. et al. The saccus vasculosus of fish is a sensor of seasonal changes in day length. Nat. Commun. 4, 1-7 (2013).

35. Koger, C. S., Teh, S. J. \& Hinton, D. E. Variations of Light and Temperature Regimes and Resulting Effects on Reproductive Parameters in Medaka (Oryzias latipes). Biol. Reprod. 61, 1287-1293 (1999).

36. Scobie, K. N. et al. Krüppel-like factor 9 is necessary for late-phase neuronal maturation in the developing dentate gyrus and during adult hippocampal neurogenesis. J. Neurosci. 29, 9875-9887 (2009).

37. Bean, N. J., Nunez, A. A. \& Conner, R. Effects of medial preoptic lesions on male mouse ultrasonic vocalizations and copulatory behavior. Brain Res. Bull. 6, 109-112 (1981).

38. De Jonge, F. H. et al. Lesions of the SDN-POA inhibit sexual behavior of male wistar rats. Brain Res. Bull. 23, 483-492 (1989).

39. Lewis, J. E. et al. Thyroid hormone and vitamin D regulate VGF expression and promoter activity. J. Mol. Endocrinol. 56, 123-134 (2016).

\section{Figures}




\section{Figure 1}

Design and results of the mating test using naïve males. (A) (B) Outline of the behavioral experiment. (A) 10 (naïve) and 11 (experienced) males were used for the study. Male and female pairs were fixed during the experiment and the experiment was carried out for 7 consecutive days. (B) Experimental procedure. The male was separated a day before the experiment and released to the female the next morning. Their mating behavior was recorded for more than 10 min and analyzed. (C) Behavioral test results. Left and right panels show the transition of the latency to mate in naïve and experienced males, respectively. The latency significantly decreased in naïve males depending on the number of matings for 5 days, but not in experienced males. Each dot represents the results of each individual. The boxplot indicates median (the center line), minimum, maximum, first quartile, third quartile, and outlier points. $P$-value shows the results of the post hoc test with Tukey's adjustment method in a generalized linear mixed model (gamma distribution, log link function).

\section{Figure 2}

Change in the courtship behavior, latency to the first courtship display (A), and the number of courtship displays (B) and the latency to mate from the first courtship display (C). Each dot represents the results of each individual. The boxplot indicates median (the center line), minimum, maximum, first quartile, third quartile and outlier points. $P$-value shows the results of the post hoc test with Tukey's adjustment method in a generalized linear mixed model (gamma distribution ( $A$ and $C$ ) and Poisson distribution (B), log link function).

\section{Figure 3}

Effect of the familiarization (repeated matings with the same partner) on mating behavior of naïve males. (A) Outline of the behavioral experiment. Each group contained 8 males in this study (fixed and swapped groups). (B) Latency to mate (left: fixed group, right: swapped group). The latency to mate significantly decreased only in the fixed group, and not in the swapped group. (C) Latency to the first courtship display (left: fixed group, right: swapped group). The latency significantly decreased only in the fixed group, and not in the swapped group. (D) Latency to mate from the first courtship. Each dot represents the results of each individual. The boxplot indicates median (the center line), minimum, maximum, first quartile, third quartile and outlier points. $P$-value shows the results of the post hoc test with Tukey's adjustment method in a generalized linear mixed model (gamma distribution, log link function).

\section{Figure 4}


Possible model of the effect of the first mating experience in male medaka. In this study, we found that 3 genes (tshba, dio2, klf9) were upregulated after the first mating experience. tshba encodes thyroid stimulating hormone, which activates inactivated thyroid hormone (T4). DIO2, which is an enzyme that converts T4 to activated thyroid hormone T3. KLF9 is expressed depending on the T3 level and contributes to spine maturation. The first mating experience may lead to maturation of male mating behavior via neural maturation depending on the thyroid hormone system in the male medaka brain.

\section{Supplementary Files}

This is a list of supplementary files associated with this preprint. Click to download.

- Supplementalinformationmd2022.pdf 